\title{
Estabelecimento de um plano de manutenção baseado em análises quantitativas no contexto da MCC em um cenário de produção JIT
}

\author{
Angélica Alebrant Mendes ${ }^{\mathrm{a} *}$, José Luis Duarte Ribeiro ${ }^{\mathrm{b}}$ \\ a*angelalebrant@yahoo.com.br, UFRGS, Brasil \\ bribeiro@producao.ufrgs.br, UFRGS, Brasil
}

\begin{abstract}
Resumo
Este artigo apresenta um método para desenvolver análises quantitativas que orientem a revisão ou elaboração de um plano de manutenção de equipamentos em um cenário de produção just in time. 0 método proposto contempla: i) Identificar os conjuntos que influenciam a confiabilidade; ii) Levantar taxas de falhas; iii) Classificar os conjuntos quanto ao efeito de suas falhas; iv) Levantar parâmetros de demanda da linha; v) ldentificar as distribuições de probabilidade da demanda da linha e dos tempos de bom funcionamento e tempos de reparo dos conjuntos; vi) Simular a produção/manutenção utilizando o método de Monte Carlo; vii) Realizar uma análise de sensibilidade a eventuais variações na demanda, $M T B F$ e $M T T R$; e viii) Estabelecer a estratégia de manutenção e intervalos entre manutenções preventivas. A aplicação do método é ilustrada através de um estudo real realizado em uma linha de rotulagem e enchimento de galões de uma empresa do setor de tintas e corantes. A aplicação do método permitiu identificar com clareza os conjuntos e subconjuntos críticos frente ao cenário produtivo em questão.
\end{abstract}

Palavras-chave

Manutenção centrada em confiabilidade. Simulação de Monte Carlo. Sistema just in time. Plano de manutenção.

\section{Introdução}

0 cenário atual, economicamente globalizado e altamente competitivo, exige que as empresas visualizem a manutenção como uma função estratégica para aumentar a produtividade. Visto que a produtividade está diretamente relacionada à redução dos custos e pode propiciar aumento de faturamento, melhorias na confiabilidade e disponibilidade dos equipamentos produtivos podem contribuir significativamente para aumentar a competitividade das organizações (SANTOS; COLOSIMO; MOTTA, 2007; MÁRQUEZ et al., 2009; PINTO; XAVIER, 2001).

Tradicionalmente, a manutenção é planejada utilizando a experiência dos funcionários envolvidos e a orientação contida nos manuais dos fabricantes dos equipamentos (LAFRAIA, 2001; RAUSAND, 1998; MENDES; RIBEIRO, 2011). Sabe-se que grande parte dos funcionários acredita na premissa de que as falhas ocorrem devido ao desgaste no tempo (WILMETH; USREY, 2000). Contudo, estudos feitos na aviação determinaram que apenas $11 \%$ dos componentes falham devido a desgaste e fadiga (LAFRAIA, 2001; WILMETH; USREY, 2000). Paralelamente, no que concerne às orientações contidas nos manuais dos fabricantes, corre-se o risco dessas recomendações não serem baseadas em dados reais, pois alguns fabricantes de equipamentos, a fim de maximizar vendas de componentes ou minimizar sua responsabilidade, orientam intervalos curtos de revisão e substituição (LAFRAIA, 2001; RAUSAND, 1998).

Para Farrero, Tarrés e Losilla (2002), a otimização da política de manutenção requer uma combinação balanceada entre manutenções preventivas, preditivas e corretivas. Sendo que a determinação do tipo de manutenção e do intervalo entre manutenções de cada item depende do comportamento de sua taxa de falhas e do custo de cada falha. Assim, a utilização de dados quantitativos tem grande importância na elaboração de planos de manutenção mais eficazes 
(RAUSAND, 1998; FARRERO; TARRÉS; LOSILLA, 2002; NGUYEN; BRAMMER; BAGAJEWICZ, 2008; SELLITTO, 2005; LAFRAIA, 2001).

Atualmente, o grande desafio para o planejamento da manutenção é exatamente a definição de quando e que tipo de intervenção deve ser feita em determinado equipamento. Apesar de existir farto material sobre confiabilidade de produtos, na perspectiva dos fabricantes, a discussão da aplicação da confiabilidade em itens em operação, na perspectiva da manutenção, ainda é escassa na literatura (SANTOS; COLOSIMO; MOTTA, 2007).

Rao, Srikrishna e Yadava (1996) justificam que abordagens qualitativas têm sido preferidas às abordagens quantitativas devido à falta de dados históricos nas plantas e de métodos estatísticos adequados para interpretar esses dados. Rausand (1998) complementa afirmando que os artigos sobre otimização da manutenção são escritos por pesquisadores matemáticos ou estatísticos que utilizam uma linguagem incompreensivel e propõem modelos de complexidade muito além do aceitável na prática da manutenção.

Dessa forma, o objetivo deste trabalho é desenvolver análises quantitativas que orientem a revisão ou elaboração de um plano de manutenção de equipamentos em um cenário de produção just in time. Cenários just in time (JIT) se caracterizam por estoques reduzidos (ou nulos) e a necessidade de atender a programação da produção no próprio turno de trabalho. Assim, nesses cenários, o que está programado para o dia d deve ser entregue no dia $d$ e não deve ser postergado para $d+1$.

Cenários just in time têm sido largamente aplicados com o objetivo de reduzir custos de estoque de matérias-primas, produtos semi-acabados e produtos acabados. Contudo, o bom desempenho desse tipo de sistema requer, além da eliminação de itens defeituosos fluindo no processo, uma produção equilibrada ao longo do tempo. Essas premissas só podem ser alcançadas através de uma manutenção eficiente, que elimine a ocorrência de defeitos gerados pelo processo nos produtos e que evite a ocorrência de falhas inesperadas nos equipamentos, a fim de manter um fluxo estável de produtos no processo.

A utilização do método de simulação de Monte Carlo para modelar a produção/manutenção em cenários de produção just in time permite a inclusão da variabilidade, natural do sistema, na simulação. Essa simulação possibilita a análise da resposta do sistema quanto às horas de parada de máquina e horas extras para atender a demanda diária, considerando possíveis variações de demanda MTBFe MTTR. Estes resultados auxiliam a empresa na tomada de decisão quanto à necessidade e ao tipo de investimento em manutenção que melhor atenderia ao cenário de demanda projetado.

A elaboração de planos de manutenção baseados em análises quantitativas é essencial para a compreensão do tipo e do intervalo de manutenção mais adequado ao comportamento da taxa de falhas de cada equipamento. Assim, é possível definir a melhor estratégia de manutenção para cada equipamento, evitando a aplicação de atividades de manutenção desnecessárias ou ineficazes e agregando atividades que efetivamente contribuem para o aumento da disponibilidade dos equipamentos e redução de custos da empresa. Vale ressaltar que questões qualitativas, como condições de acesso aos equipamentos, recomendações apresentadas em normas e regulamentos, treinamento do pessoal de manutenção e outras atividades programadas para o mesmo equipamento também devem ser consideradas.

Este artigo está estruturado em cinco seções. $\mathrm{Na}$ seção 2 é apresentado o referencial teórico sobre os métodos de elaboração de planos de manutenção e seus elementos. Na seção 3 são apresentados os procedimentos metodológicos utilizados para realizar o trabalho e solucionar o problema de pesquisa. Os resultados obtidos, bem como sua discussão são apresentados na seção 4. Por fim, na seção 5, são apresentadas as conclusões referentes ao estudo realizado.

\section{Referencial teórico}

A literatura indica que os principais elementos a serem definidos em um plano de manutenção para um equipamento são: i) a estratégia de manutenção para cada sistema ou componente; e ii) o intervalo de tempo entre manutenções (BLO0M, 2006; RAUSAND, 1998; SELLITTO, 2005).

A escolha da melhor estratégia de manutenção envolve, além da identificação do comportamento da taxa de falhas do componente no decorrer do tempo, a verificação das atividades de manutenção aplicáveis e a criticidade da falha, considerando aspectos associados à economia, segurança e meio ambiente (BLOOM, 2006; FOGLIATTO; RIBEIRO, 2009; NGUYEN; BRAMMER; BAGAJEWICZ, 2008).

A melhor estratégia de manutenção usualmente é a manutenção preditiva, contudo, se seus custos são proibitivos ou se um método para monitoramento da condição do equipamento ainda não foi desenvolvido, é indicada a manutenção preventiva baseada no tempo. Se a manutenção preventiva não é aplicável, deve-se analisar se a falha pode ser tolerada. Se a falha não pode ser tolerada, é necessário um "redesign" 
do equipamento, se ela é tolerável, deve-se aplicar a manutenção corretiva e adotar procedimentos de procura por falhas, buscando identificar falhas escondidas (WILMETH; URSEY, 2000; FOGLIATTO; RIBEIRO, 2009; PINTO; XAVIER, 2001).

Com base em dados qualitativos das falhas dos componentes, diversos autores apresentam diagramas de decisão para a determinação da melhor estratégia de manutenção (BLOOM, 2006; FOGLIATTO; RIBEIRO, 2009; PINTO; XAVIER, 2001; RAUSAND, 1998; RAO; SRIKRISHNA; YADAVA, 1996; DESHPANDE; MODAK, 2002). Esses diagramas relacionam informações relativas à evidência da falha, suas consequências, previsão de desgaste do componente no tempo e aplicabilidade da manutenção.

Bevilacqua, Braglia e Gabrielli (2000) propõem uma metodologia baseada na integração entre FMECA modificada e simulação de Monte Carlo para determinar o tipo de manutenção mais apropriado para cada equipamento. 0 número de prioridade de risco $(R P M)$ é definido através da soma do produto de pesos de seis parâmetros (segurança, importância da máquina para o processo, custos de manutenção, frequência de falhas, tempo de reparo e condições de operação) pelo desempenho da máquina nesses parâmetros. Os pesos estipulados para cada parâmetro são analisados quanto à sua robustez através da simulação de Monte Carlo.

Sellitto (2005) e Lafraia (2001) definem a melhor estratégia de manutenção com base em dados estatísticos das falhas dos componentes. Através da determinação dos parâmetros da distribuição de Weibull, obtida através do ajuste dos dados de falhas, é possível identificar se o componente encontra-se no periodo de falhas prematuras (parâmetro de forma $<1$ ), maturidade (falhas aleatórias, parâmetro de forma $=1$ ) ou desgaste (parâmetro de forma $>1$ ). As estratégias de manutenção aconselhadas são: i) fase de falhas prematuras - manutenção corretiva; ii) fase de maturidade - manutenção preditiva e gestão de boas práticas de manutenção; iii) fase de desgaste - manutenção preventiva.

Para a determinação do intervalo de tempo entre manutenções, Bloom (2006) recomenda a utilização da experiência e do bom senso, visto que, além do histórico de falhas, é necessário considerar fatores como: recomendações dos fabricantes, orientação de normas e regulamentos, paradas programadas, condições de acesso ao componente e outras atividades de manutenção programadas para o mesmo componente. 0 autor ainda destaca a necessidade de revisões nos intervalos de manutenção. Essas devem ser baseadas no feedback constante dos operadores da manutenção quanto à condição do equipamento no momento da execução da atividade.
Rao, Srikrishna e Yadava (1996) apresentam um algoritmo para a otimização do intervalo entre manutenções baseado no menor custo total de manutenções preventivas e corretivas. Santos, Colosimo e Motta (2007) propõem um modelo para determinação do tempo ótimo entre manutenções preventivas também baseado no custo ótimo de manutenção, porém utilizando o processo de Poisson homogêneo e não homogêneo.

Sellitto (2005) e Lafraia (2001) obtêm o intervalo ótimo de intervenção através das relações de custo entre intervenções de emergência e preventiva $\left(R_{\mathrm{EP}}\right)$. Lafraia (2001) ainda apresenta um método para determinação do intervalo ótimo de intervenção baseado na maximização da disponibilidade média do componente. Supondo que a manutenção preventiva seja perfeita, recuperando a taxa de falha ao seu valor inicial, para taxas de falha $(\lambda)$ linearmente crescentes, decrescentes ou constantes no tempo, o autor demonstra que a frequência ótima de manutenção preventiva pode ser obtida pela Equação 1:

$\lambda p^{*}=\sqrt{\frac{\alpha r \mu p}{2 \mu c}}$

onde $\alpha r$ é o aumento da taxa de falha por ano ou o coeficiente da equação linear da taxa de falha, $\mu p$ é a taxa de ação de manutenção preventiva, $\mu c$ é a taxa de reparo de manutenção corretiva.

Em resumo, as análises quantitativas que podem dar suporte ao planejamento da manutenção, no âmbito da MCC, seriam: para a definição da estratégia de manutenção mais adequada, análise estatística do comportamento da taxa de falhas do sistema, FMECA modificada, e simulação de Monte Carlo; para a determinação do melhor intervalo entre manutenções têm-se análises do custo ótimo de manutenção e maximização da disponibilidade do sistema.

\section{Método de trabalho}

Este trabalho foi realizado nas seguintes etapas: i) Identificação dos conjuntos ou subconjuntos que influenciam a confiabilidade dos equipamentos a serem estudados; ii) Levantamento das taxas de falhas dos conjuntos ou subconjuntos identificados; iii) Classificação dos conjuntos ou subconjuntos quanto ao efeito de suas falhas (tempos de reparo, tempos de bom funcionamento e disponibilidade); iv) Levantamento dos parâmetros de demanda da linha; v) Identificação das distribuições de probabilidade da demanda da linha, dos tempos de bom funcionamento e dos tempos de reparo dos subconjuntos; vi) Simulação da produção/manutenção a fim de definir, através de métodos estocásticos, a probabilidade de que a 
linha não atenda a demanda diária de produção; vii) Realização de uma análise de sensibilidade das horas extras e tempos de parada de máquinas a eventuais variações na demanda, MTBF e MTTR; e iv) Estabelecimento da estratégia de manutenção e dos intervalos entre manutenções preventivas.

0 método idealizado foi aplicado na filial gaúcha de uma empresa multinacional do setor de tintas e corantes. Escolheu-se a linha de rotulagem e enchimento de galões, chamada Packer, devido a sua criticidade em termos de demanda e número de chamados de manutenção. A equipe da manutenção da empresa, juntamente com a equipe de operação da linha, identificaram os sistemas e componentes significativos a serem analisados. Foram identificados 6 conjuntos e 17 subconjuntos.

0 levantamento das taxas de falha foi feito através da coleta de dados de manutenção existentes nas ordens de serviço da empresa dos anos de 2008 e 2009 (descrição da falha, tempo de reparo e data de ocorrência). Os dados foram organizados e revisados pela equipe de manutenção, pois muitas ordens de serviço não apresentavam descrições claras e relevantes sobre o serviço de manutenção executado. Foram identificados os modos de falha e o conjunto e subconjunto referentes a cada falha.

Na sequência, foram identificadas as possíveis causas e consequências de cada falha e foram calculados os tempos médios de reparo (MTTR), tempos médios de bom funcionamento (MTBF) e a disponibilidade de cada subconjunto. Para os subconjuntos que não apresentavam registro da ocorrência de falha em todo o período analisado foi considerada disponibilidade $=1,0$.

Para verificar o modelo de distribuição de probabilidade que melhor se ajustava aos dados de tempos de bom funcionamento de cada subconjunto foram realizados testes estatísticos no software PROCONF. 0 modelo de distribuição de Weibull mostrou-se adequado para todos os subconjuntos, sendo que os parâmetros de forma sempre resultaram próximo de 1,0. Sendo assim, optou-se pela distribuição exponencial para modelar os subconjuntos (a distribuição exponencial é um caso particular da distribuição de Weibull em que a taxa de falha é constante e o parâmetro de forma é igual a 1,0). As Figuras 1 e 2 ilustram as saídas gráficas dessas análises para dois subconjuntos. Vale observar que uma taxa de falha aproximadamente constante é esperada para esses subconjuntos, uma vez que, conforme o relato das equipes de operação e manutenção, eles se encontram na fase de maturidade (fase intermediária da tradicional curva da banheira, onde a taxa de falha é aproximadamente constante).
Quanto aos tempos de reparo, o mesmo comportamento foi observado. Os tempos de reparo dos subconjuntos seguem aproximadamente a distribuição exponencial, a qual foi selecionada para representá-los. Essa constatação está de acordo com as premissas apresentadas por Sellitto (2005) e Lafraia (2001) segundo a qual reparos em equipamentos industriais são atividades com componente intelectivo e cognitivo e podem seguir as características dessa distribuição. Segundo Sellitto (2005), em tempos para reparo também é possível encontrar a distribuição normal, quando o reparo for uma soma de tarefas independentes e sequenciais, tal como ocorre em reformas de máquinas e em procedimentos preventivos mais complexos e intrincados, o que não reflete 0 caso em estudo.

Vale observar que a empresa não executava manutenções preventivas periódicas no equipamento em estudo. Assim, a estratégia de manutenção adotada foi essencialmente corretiva. Contudo, a empresa demonstrou a intenção de implantar a manutenção preventiva, o que motivou o presente estudo.

$\mathrm{Na}$ etapa seguinte, foram coletados dados de demanda da linha referentes às horas de operação

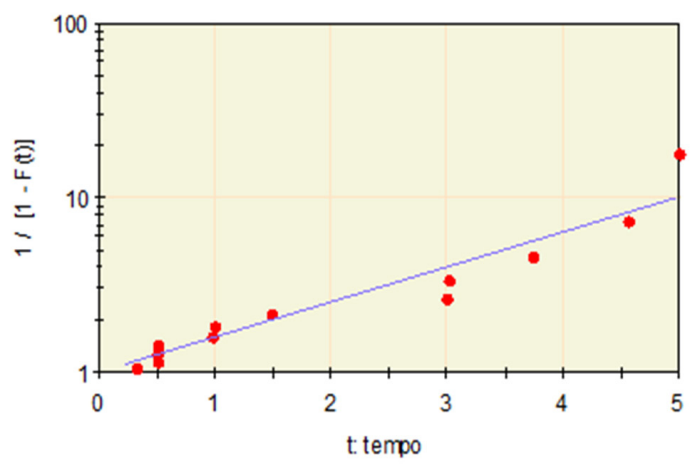

Figura 1. Papel de probabilidade da distribuição exponencial para tempos de bom funcionamento do subconjunto B6.

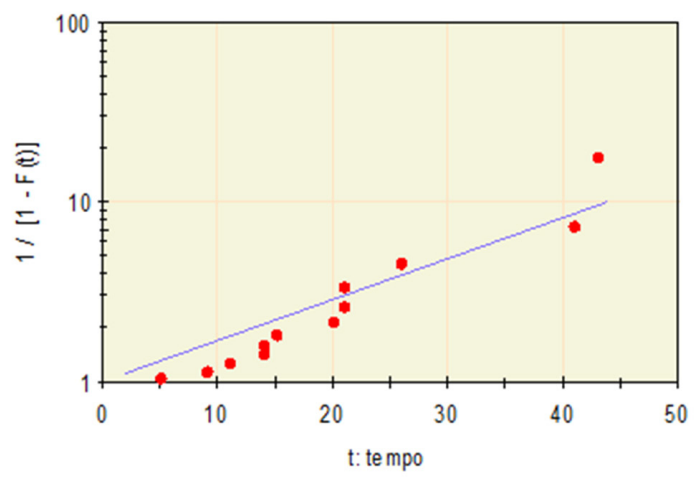

Figura 2. Papel de probabilidade da distribuição exponencial para tempos de bom funcionamento do subconjunto F20. 
diárias e mensais, no período de janeiro de 2009 a julho de 2010. Baseado nesses dados foi possível ajustar uma distribuição de probabilidade ao número de horas de trabalho por dia. Uma distribuição normal, com média 12 horas por dia e desvio padrão de 1,5 horas por dia apresentou um ajuste adequado aos dados.

Após a definição das distribuições de probabilidade apropriadas para modelar os tempos de bom funcionamento, os tempos de reparo e a demanda da linha foi desenvolvido um estudo de simulação de Monte Carlo das atividades de produção, falhas e manutenção. Essa simulação permitiu estudar, através de métodos estocásticos, a probabilidade de a linha atender a demanda diária de produção. A simulação envolveu a determinação da demanda diária da linha, com base nos dados de demanda, a identificação da ocorrência da falha, com base nos tempos de bom funcionamento, e a definição dos tempos de reparo, utilizando-se a distribuição desses.

A simulação permitiu verificar a probabilidade de a linha não atender a demanda diária de produção devido ao possível desequilíbrio entre horas de trabalho demandadas e horas disponíveis, que podem ser reduzidas em função de falhas e respectivos reparos. Vale observar que sempre que as horas demandadas de trabalho superam as horas disponíveis, a política de produção, em função do cenário JIT, exige horas extras. 0 cenário JIT vivenciado pela empresa impõe que a demanda diária deve ser atendida no dia.

Posteriormente foi realizada uma análise de sensibilidade das horas extras e horas de parada de máquina a variações na demanda, tempos de bom funcionamento e tempos de reparo. Foram gerados gráficos, representando cenários em que variaram a demanda, o $M T B F$ e o $M T T R$, permitindo a visualização do impacto dessas variáveis sobre as horas extras.

Por fim, após verificar no estudo de simulação a necessidade de melhorias na manutenção para aumentar a disponibilidade do equipamento, devido à previsão de aumento da demanda, foi estabelecida uma nova estratégia de manutenção e foram revistos os intervalos entre manutenções preventivas. Esses novos intervalos estão de acordo com a confiabilidade desejada pela empresa.

\section{Resultados e discussões}

Após a classificação em conjuntos e subconjuntos e o levantamento dos respectivos tempos de bom funcionamento e tempos de reparo, foi possivel calcular o tempo médio de reparo, o tempo médio de bom funcionamento e a disponibilidade de cada subconjunto, conforme apresentado na Tabela 1. Para os três subconjuntos que não apresentaram nenhuma falha no período analisado foram consideradas disponibilidade igual a 1,0. Nas Figuras 3, 4 e 5 são apresentados gráficos do $M T B F$, do $M T T R$ e da disponibilidade dos subconjuntos, respectivamente.

A Figura 5 mostra que os sensores de posicionamento do carrossel de rotulagem são o subconjunto que apresenta menor disponibilidade $(0,9946)$. Esse fato deve-se, como pode ser observado na Figura 3, ao pequeno tempo médio de bom funcionamento $(M T B F)$. Em média, a cada onze dias ocorre uma intervenção de manutenção nesse subconjunto. Por outro lado, a junta rotativa superior,

Tabela 1. Conjuntos e subconjuntos do sistema.

\begin{tabular}{|c|c|c|c|c|c|c|}
\hline Conjunto & $\mathrm{Cj}$ & Subconjunto & Scj & $\operatorname{MTTR}(\mathrm{h})$ & $M T B F$ (dias) & Disponibilidade \\
\hline \multirow[t]{3}{*}{ Carrossel de rotulagem } & A & Sistema motriz A & 2 & 3,389 & 57,375 & 0,9971 \\
\hline & A & Embreagem do posicionador & 3 & \multicolumn{2}{|c|}{ Não possui registro de falhas } & 1,0000 \\
\hline & A & Sensores de posicionamento & 4 & 3,532 & 11,323 & 0,9846 \\
\hline \multirow[t]{3}{*}{ Esteira transportadora } & B & $\begin{array}{l}\text { Sistema motriz B (motor, redutor } \\
\text { e acionamento elétrico) }\end{array}$ & 5 & \multicolumn{2}{|c|}{ Não possui registro de falhas } & 1,0000 \\
\hline & B & Polias, mancais, esteiras e guias & 6 & 4,106 & 23,318 & 0,9913 \\
\hline & B & $\begin{array}{l}\text { Sistema de controle de aplicação, } \\
\text { enchimento e fechamento }\end{array}$ & 7 & 2,153 & 34,000 & 0,9968 \\
\hline \multirow[t]{7}{*}{ Carrossel de enchimento } & $\mathrm{C}$ & Junta rotativa superior & 10 & 16,350 & 62,250 & 0,9870 \\
\hline & C & Bicos de enchimento & 11 & 4,178 & 96,450 & 0,9978 \\
\hline & C & Balanças/Módulos de pesagem & 12 & 5,859 & 35,297 & 0,9918 \\
\hline & C & Escovas de orientação & 13 & 5,251 & 31,375 & 0,9917 \\
\hline & $\mathrm{C}$ & Junta rotativa inferior & 17 & \multicolumn{2}{|c|}{ Não possui registro de falhas } & 1,0000 \\
\hline & C & Sistema de lavagem & 18 & 5,011 & 94,950 & 0,9974 \\
\hline & C & Sistema motriz C & 19 & 3,000 & 98,625 & 0,9985 \\
\hline \multirow[t]{2}{*}{ Largador e fechador de tampas } & $\mathrm{D}$ & Largador de tampas & 14 & 4,987 & 26,063 & 0,9905 \\
\hline & $\mathrm{D}$ & Fechador de tampas & 15 & 8,138 & 83,500 & 0,9952 \\
\hline Quadros de comando & $\mathrm{E}$ & Quadros de comando & 21 & 1,617 & 21,750 & 0,9963 \\
\hline Sistema etiquetadora & $\mathrm{F}$ & Etiquetadora & 20 & 3,419 & 19,442 & 0,9913 \\
\hline
\end{tabular}




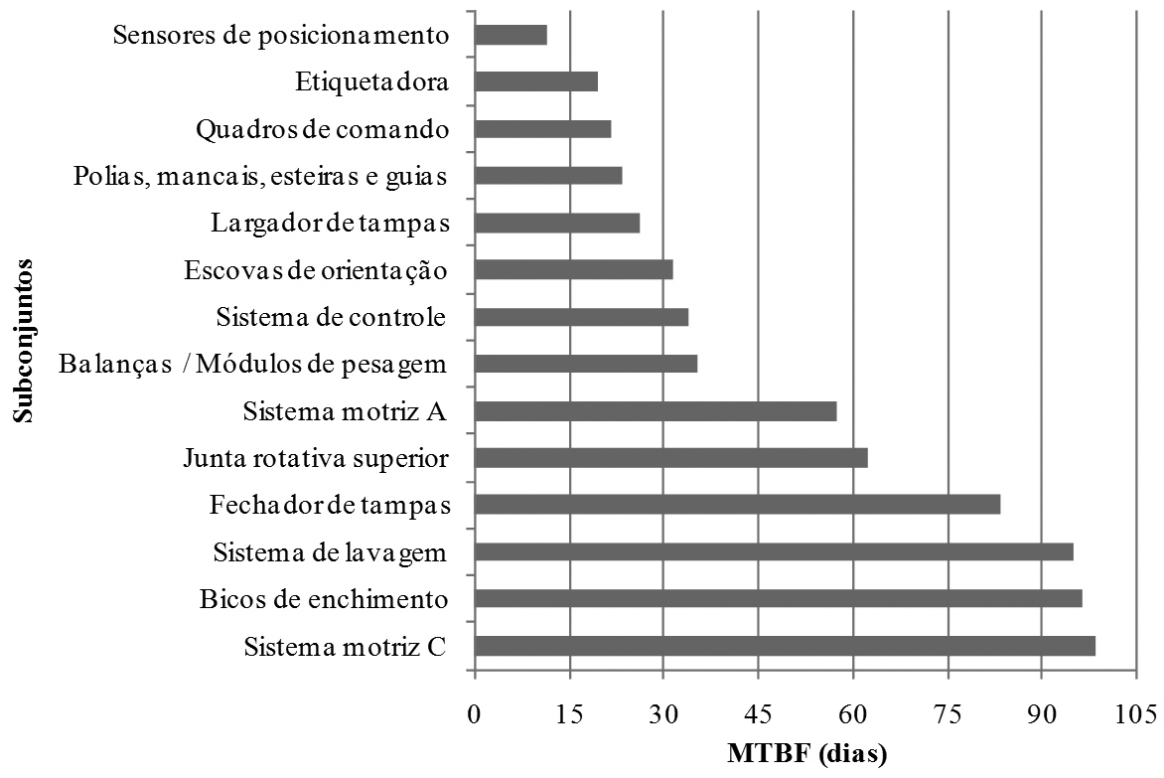

Figura 3. MTBF em dias dos subconjuntos.

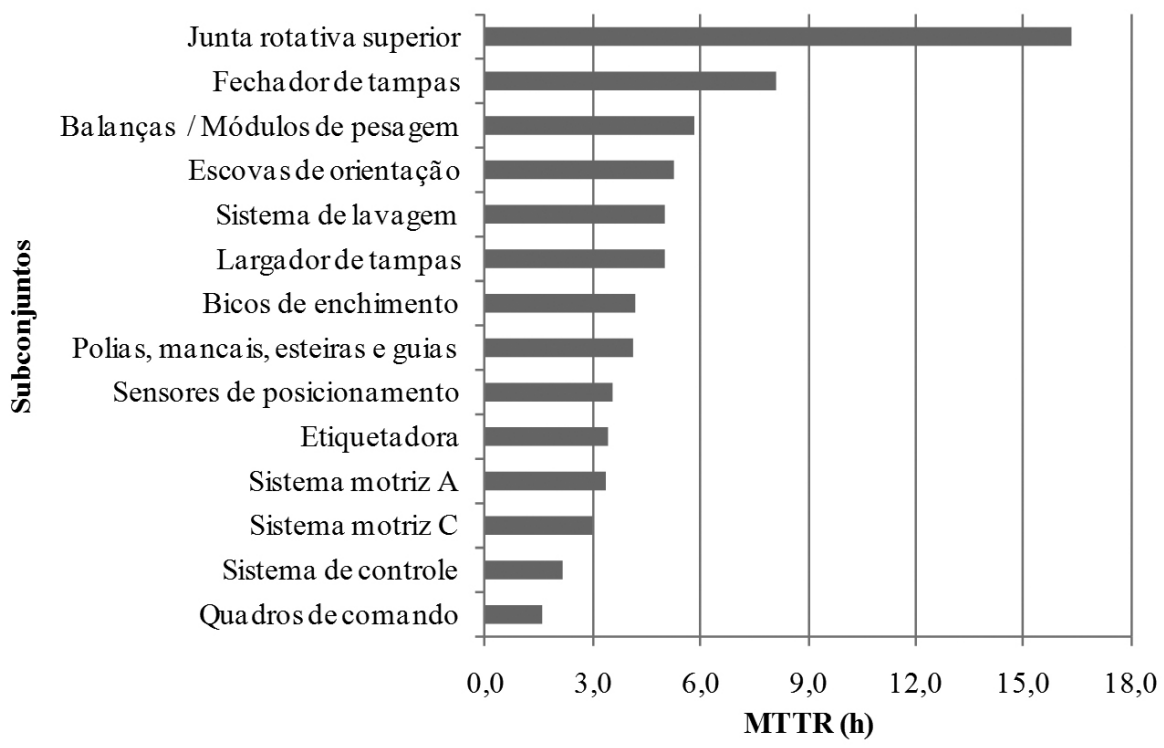

Figura 4. MTTR em horas dos subconjuntos.

que aparece na Figura 5 como o subconjunto com o segundo menor índice de disponibilidade $(0,9870)$, apresenta um tempo médio para reparo (MTTR) significativamente elevado com relação aos demais subconjuntos, sendo superior a 16 horas, conforme Figura 2. Dessa forma, observa-se serem necessárias ações para reduzir o número de ocorrências de falhas nos sensores de posicionamento e melhorar a rotina de manutenção da junta rotativa superior, a fim de aumentar a sua disponibilidade e, consequentemente, a disponibilidade da linha.
Após identificar o modelo de distribuição exponencial como aquele que melhor se ajusta aos dados de tempos de bom funcionamento e tempos de reparo dos subconjuntos, calculou-se, através do inverso do $M T B F$, a taxa de falha de cada subconjunto.

A simulação da produção, falhas e manutenção foi realizada seguindo os princípios do método de Monte Carlo. Para basear a análise em uma amostra suficientemente grande, foram simulados 1.290 dias, que equivalem a 5 anos de trabalho. 


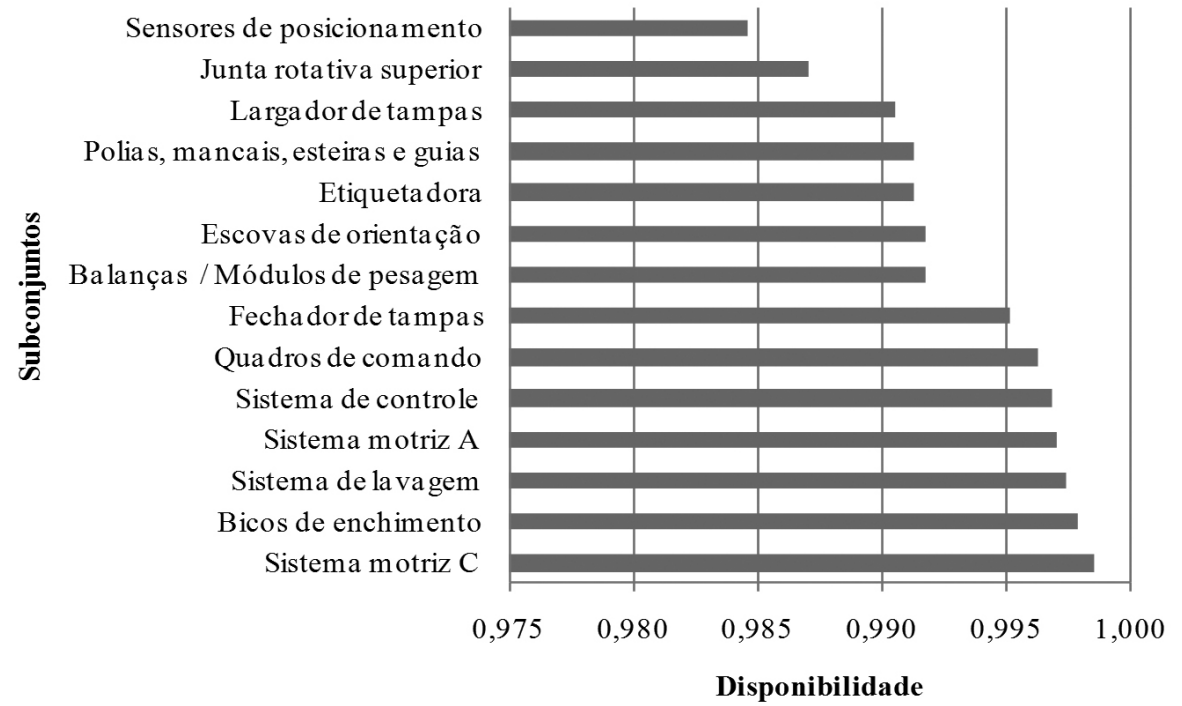

Figura 5. Disponibilidade dos subconjuntos.

Dividiu-se a simulação em quatro etapas, conforme detalhado a seguir:

Etapa 1 - Simulação da demanda diária - após verificar-se que os dados de demanda média diária da linha se ajustavam ao modelo de distribuição normal, foram gerados números aleatórios (adotados como percentis) e, utilizando-se a função normal, eles foram transformados em valores da distribuição da demanda diária;

Etapa 2 - Simulação das falhas - baseando-se na taxa de falha e respectiva distribuição de probabilidade de falha, foram identificadas a probabilidade de falha de cada subconjunto em um dia genérico. A seguir foram gerados números aleatórios: a condição de falha caracterizava-se sempre que o número aleatório gerado para um determinado subconjunto era inferior à probabilidade de falha desse subconjunto;

Etapa 3 - Simulação dos tempos de reparo (TTR) - para os subconjuntos que falharam em cada dia simulado foram gerados tempos de reparo utilizando-se as respectivas distribuições dos tempos de reparo dos subconjuntos. Isso foi feito gerando números aleatórios (adotados como percentis) e, utilizando a distribuição exponencial, eles foram transformados em valores da distribuição dos tempos de reparo dos subconjuntos envolvidos;

Etapa 4 - Simulação de horas extras - para cada dia simulado foram somados a demanda diária com os tempos de reparo dos subconjuntos que falharam naquele dia. Sempre que essa soma ultrapassou as horas de produção disponíveis houve necessidade de horas extras de trabalho. As horas extras são geradas diariamente, visto que o sistema just in time busca o atendimento da programação da produção no dia. Nas situações em que a soma das horas disponíveis mais horas extras ultrapassaram 24 horas, as horas extras excedentes foram contabilizadas no dia útil subsequente (usualmente, nesses casos, existe alguma multa contratual infligida ao fabricante, mas a quantificação de eventuais multas não foi objeto deste estudo).

Devido à baixa ocorrência de horas extras na linha não foi considerada a possibilidade de falha durante a realização das horas extras. A Figura 6 apresenta um fluxograma da simulação supondo distribuições de demanda, tempos de falha e tempos de reparo dos componentes conhecidas.

Assim, as variáveis de controle ou de entrada para a simulação são a demanda média, prevista em horas por dia, o desvio padrão da demanda, além do MTBF e o MTTR em horas para todos os subconjuntos do sistema. Como resultados da simulação têm-se a estimativa de horas de máquina parada por mês e a estimativa de horas extras necessárias para atender a demanda mensal. Caso a soma das horas de demanda e horas de reparo de determinado dia seja superior a 17 horas (horas regulares trabalhadas diariamente, produção operando em dois turnos), haverá a necessidade de horas extras, caso contrário, a demanda será suprida durante o horário normal de trabalho. A Figura 7 apresenta resumidamente a planilha de simulação.

A simulação permite estimar a quantidade de horas extras que seriam necessárias para suprir determinada demanda diária a partir de valores pré-estabelecidos de $M T B F$ e MTTR do sistema. 0 método de Monte 


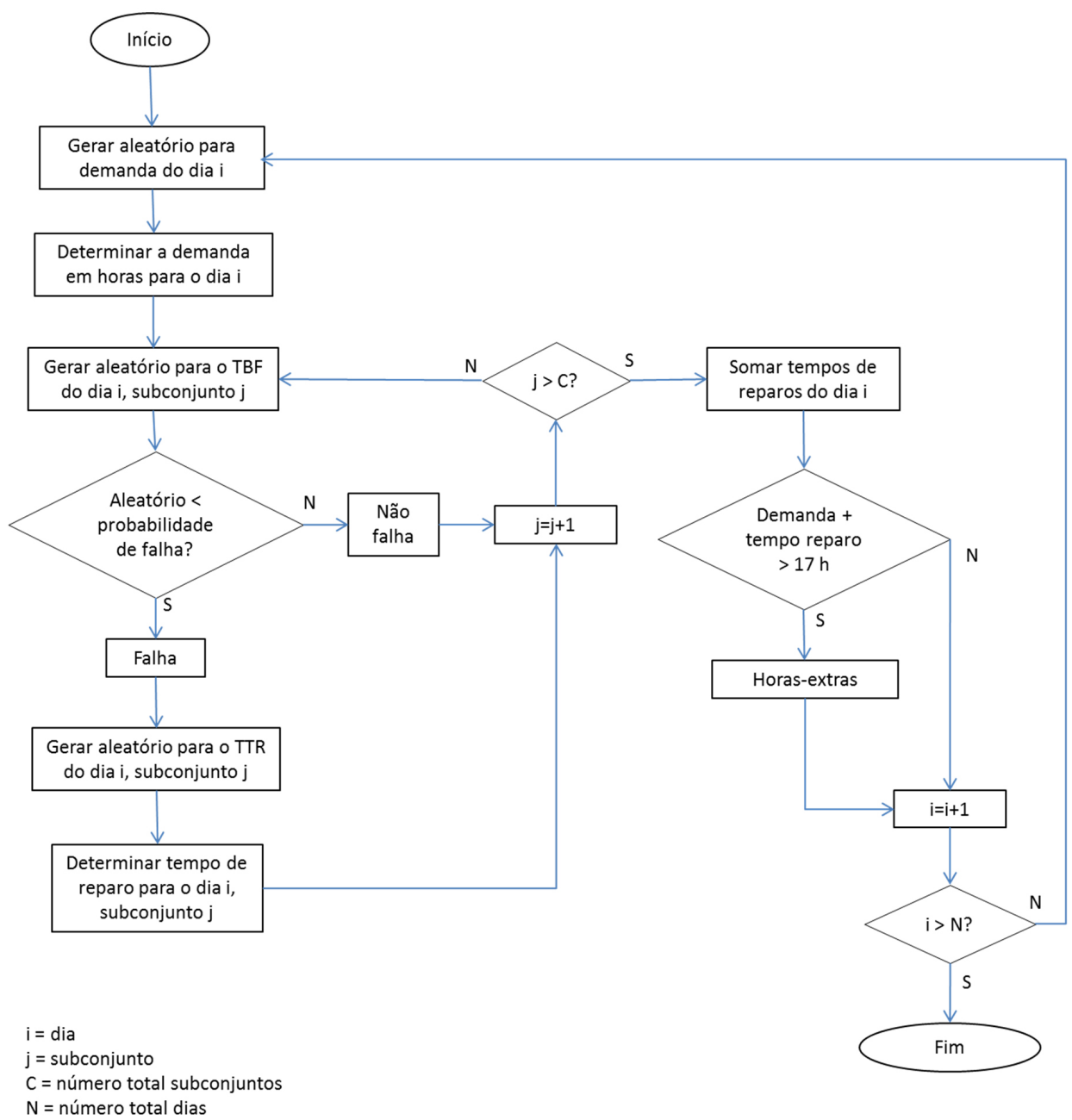

Figura 6. Fluxograma da simulação.

Carlo possibilita a inclusão da variabilidade, inerente às variáveis estocásticas envolvidas neste estudo, na simulação, o que gera resultados mais próximos da realidade do sistema em estudo.

A fim de facilitar a visualização dos resultados gerados pela simulação, foi realizada uma análise de sensibilidade das horas extras e horas de parada de máquina a variações na demanda, MTBF e MTTR. Foram gerados vários cenários com variações de demanda, MTBF e MTTR, os quais são apresentados na Figura 8. Cada gráfico corresponde à simulação de um valor de MTTR, sendo que cada curva no gráfico representa um valor de $M T B F$. Cada ponto em cada gráfico ilustra a necessidade de horas extras para um cenário gerado pela combinação de valores de demanda, MTBF e MTTR.

Vale observar que a resposta a variações na demanda era o interesse principal da empresa em estudo, uma vez que, em função do cenário econômico, a gerência prevê que a demanda deve crescer no próximo ano. Variações no $M T B F$ seriam resultado de alterações na política atual de manutenção preventiva e preditiva. Por exemplo, intensificar as atividades de manutenção preditiva e preventiva resultará tipicamente em um aumento no MTBF. Variações no MTTR seriam resultado de alterações na capacitação e nos recursos da equipe de manutenção. Por exemplo, reforço na capacitação da equipe de manutenção e melhorias nas ferramentas e estoque de peças de reposição resultarão tipicamente em uma redução no MTTR.

Os gráficos apresentados demonstram que, à medida que a demanda cresce, a quantidade de horas extras necessárias também aumenta. Ainda que a demanda média seja inferior à capacidade produtiva disponível, em casos onde ela é próxima ao valor da 


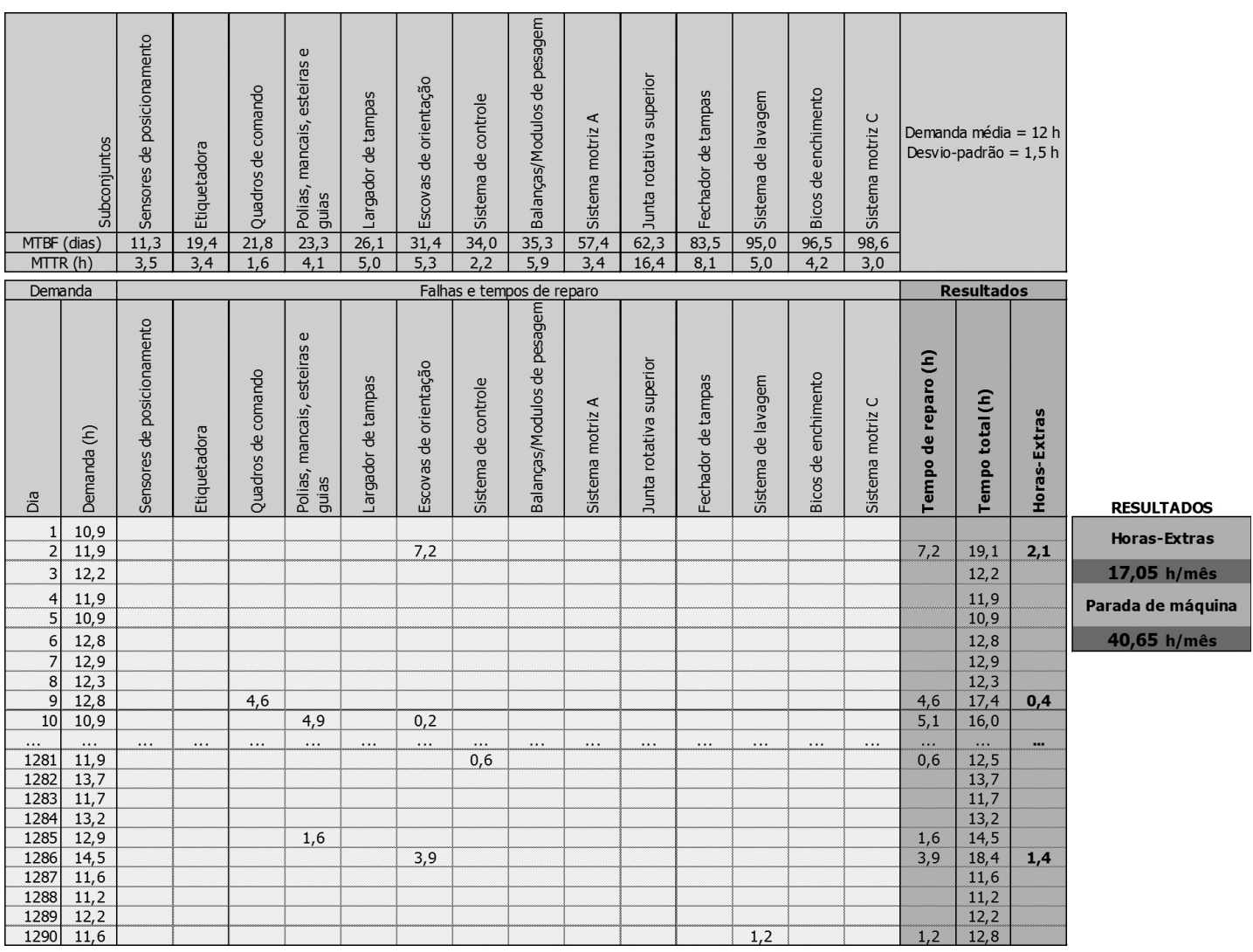

Figura 7. Resumo da planilha de simulação.

capacidade produtiva o seu perfil estocástico produz dias de demanda superior à capacidade, o que gera a necessidade de horas extras. Tal fato é agravado quando há ocorrência de falhas que necessitam reparos emergenciais e causam interrupção da produção.

Os gráficos também permitem visualizar o comportamento da necessidade de horas extras variando-se o $M T B F$ e o MTTR. Quanto maior é o $M T B F$, menor é o número de falhas do sistema e, consequentemente, menor é o tempo gasto em atividades de reparo, o que permite que a capacidade produtiva seja melhor aproveitada. Do mesmo modo, quanto menor for o MTTR, menor será o tempo gasto em manutenções corretivas, o que aumenta a disponibilidade do sistema.

Ainda que MTBFe MTTR possuam o mesmo efeito sobre a capacidade produtiva do sistema, ambos são independentes. MTBF está associado ao número de falhas e MTTR se refere ao tempo gasto para reparar cada falha. Pode-se verificar, através dos gráficos, que variações percentuais no MTTR produzem maior impacto nas horas extras que variações nos mesmos percentuais no MTBF. Logo, no caso do equipamento estudado, é mais eficaz, para reduzir as horas extras mensais, concentrar esforços na redução do MTTR, através de ações de melhoria na gestão e nas práticas de manutenção empregadas no sistema. Observa-se também que, à medida que o MTTR diminui, ações para aumento do $M T B F$ se tornam menos eficazes na redução das horas extras mensais.

A simulação permite que a empresa defina, de acordo com o seu cenário, se investimentos em melhorias na manutenção são vantajosos e qual foco de melhoria (no subconjunto ou nas práticas de manutenção) seria mais eficiente para aumentar a disponibilidade da linha produtiva. Casos onde a demanda é pequena, ainda que a disponibilidade seja baixa, não justificam investimentos em melhoria da manutenção, visto que a necessidade de horas extras para atender a demanda será baixa. Por outro lado, casos em que a demanda é elevada exigem melhor desempenho da manutenção.

Diante do cenário de crescimento de demanda previsto pela empresa, a simulação demonstrou que o sistema atual não possui capacidade suficiente para atender essa demanda diária de forma satisfatória. 0 atendimento da demanda requer uma quantidade muito alta de horas extras, o que aumenta consideravelmente 


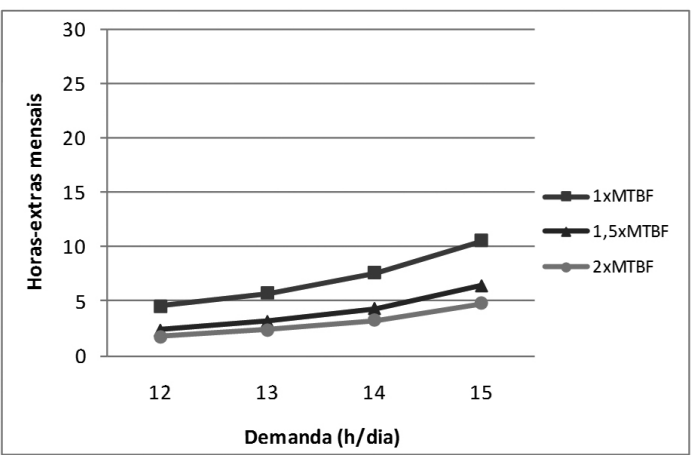

a) $0,5 \times M T T R$ original

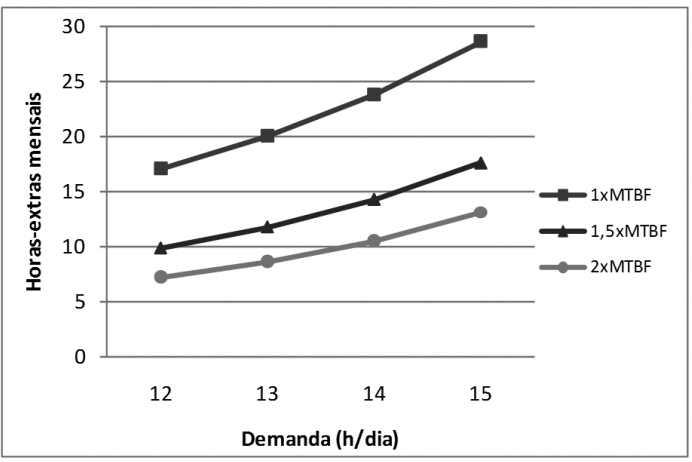

c) $1 \times$ MTTR original

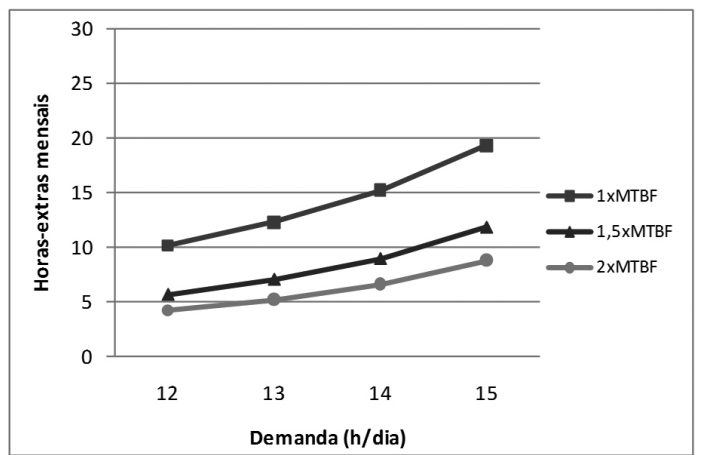

b) 0,75 xMTTR original

Figura 8. Análise de sensibilidade das horas extras a variações na demanda, MTTR e MTBF.

os custos de produção. Por exemplo, para uma previsão de demanda de 15 horas por dia, seriam necessárias cerca de 29 horas extras no mês. Buscando reduzir esse número de horas extras mensais através de melhorias na gestão da manutenção, foram determinados novos intervalos entre manutenções para os subconjuntos.

Os intervalos de manutenção para cada subconjunto foram definidos em consenso com a equipe de manutenção e operação. Tendo em vista os custos de parada de linha e os custos de manutenção, a equipe escolheu adotar uma confiabilidade de $80 \%$, ou seja, a equipe aceita uma probabilidade de $20 \%$ de que a falha ocorra antes da visita de manutenção preventiva. 1solando-se a variável tempo $(t)$ da fórmula da confiabilidade para distribuição exponencial (Equação 2), obtém-se o tempo calculado para a realização de visitas periódicas de manutenção em cada subconjunto.

para $\mathrm{R}(\mathrm{t})=0,8$ tem-se: $t=M T B F \times[-i n(0,8)]$

Contudo, para facilitar a programação da manutenção, os subconjuntos com tempos para manutenção semelhantes foram agrupados e um tempo médio comum para a realização dessas visitas foi recomendado. A Tabela 2 apresenta os tempos para manutenção calculados e recomendados para os subconjuntos.

Recomenda-se a realização de visitas periódicas para manutenção: três vezes por semana para os sensores de posicionamento; a cada 7 dias para etiquetadora, quadros de comando, polias, mancais, esteiras e guias, largador de tampas, escovas de orientação, sistema de controle de aplicação, enchimento e fechamento e balanças/módulos de pesagem; a cada 14 dias para sistema motriz A e junta rotativa superior; a cada 21 dias para fechador de tampas, sistema de lavagem, bicos de enchimento e sistema motriz C; a cada 180 dias para embreagem do posicionador, sistema motriz B e junta rotativa inferior. É importante ressaltar que os tempos recomendados para manutenção são para visitas de verificação das condições de operação do subconjunto, conduzindo, quando necessário, à realização de lubrificações, limpezas, reapertos e substituições de componentes.

A definição das atividades de manutenção mais adequadas para cada subconjunto foi baseada na experiência dos responsáveis pela manutenção e dos operadores da linha, bem como na recomendação nos manuais dos equipamentos. Contudo, a periodicidade 
Tabela 2. Tempos em dias calculados e recomendados para manutenção.

\begin{tabular}{|c|c|c|c|c|c|}
\hline Conjunto & & Subconjunto & $\begin{array}{l}\text { Tempo } \\
\text { calculado }\end{array}$ & $\begin{array}{c}\text { Tempo } \\
\text { recomendado }\end{array}$ & Tempo recomendado \\
\hline A & 4 & Sensores de posicionamento & 2,5 & 2,8 & Três vezes por semana \\
\hline $\mathrm{F}$ & 20 & Etiquetadora & 4,3 & 7 & Uma vez por semana \\
\hline $\mathrm{E}$ & 21 & Quadros de comando & 4,9 & 7 & \\
\hline $\mathrm{B}$ & 6 & Polias, mancais, esteiras e guias & 5,2 & 7 & \\
\hline $\mathrm{D}$ & 14 & Largador de tampas & 5,8 & 7 & \\
\hline $\mathrm{C}$ & 13 & Escovas de orientação & 7,0 & 7 & \\
\hline B & 7 & Sistema de controle de aplicação, enchimento e fechamento & 7,6 & 7 & \\
\hline $\mathrm{C}$ & 12 & Balanças /Módulos de pesagem & 7,9 & 7 & \\
\hline A & 2 & Sistema motriz A & 12,8 & 14 & Uma vez a cada duas \\
\hline $\mathrm{C}$ & 10 & Junta rotativa superior & 13,9 & 14 & semanas \\
\hline $\mathrm{D}$ & 15 & Fechador de tampas & 18,6 & 21 & Uma vez a cada três \\
\hline $\mathrm{C}$ & 18 & Sistema de lavagem & 21,2 & 21 & semanas \\
\hline C & 11 & Bicos de enchimento & 21,5 & 21 & \\
\hline $\mathrm{C}$ & 19 & Sistema motriz C & 22,0 & 21 & \\
\hline A & 3 & Embreagem do posicionador & 178,5 & 180 & Uma vez por semestre \\
\hline B & 5 & Sistema motriz B & 178,5 & 180 & \\
\hline $\mathrm{C}$ & 17 & Junta rotativa inferior & 178,5 & 180 & \\
\hline
\end{tabular}

dessas manutenções levou em consideração o tempo recomendado de manutenção definido anteriormente. Foram elaboradas folhas de verificação contendo informações sobre as atividades e periodicidade da manutenção para os operadores, no caso de manutenção autônoma, e para os técnicos de manutenção, no caso de manutenção não autônoma.

\section{Conclusão}

0 objetivo principal deste trabalho foi desenvolver análises quantitativas que orientassem a revisão ou elaboração de um plano de manutenção de equipamentos em um cenário de produção just in time. Cenários just in time se caracterizam por estoques reduzidos (ou nulos) e a necessidade de atender a programação da produção no próprio turno de trabalho.

Após a identificação dos conjuntos e subconjuntos que influenciam a confiabilidade dos equipamentos estudados, foram levantadas suas taxas de falhas, tempos de bom funcionamento, tempos de reparo e disponibilidade. A seguir, foram obtidos dados de demanda da linha de produção e sua distribuição de probabilidade, bem como as distribuições de probabilidade dos tempos de bom funcionamento e tempos de reparo. Posteriormente, foi realizada uma simulação da produção, falhas e manutenção, utilizando-se o método de Monte Carlo, a fim de definir, através de métodos estocásticos, a probabilidade de a linha não atender a demanda diária de produção. Na sequência foi realizada uma análise de sensibilidade das horas extras e tempos de parada de máquinas a eventuais variações na demanda, MTBF e MTTR. Através dos resultados da simulação do cenário de crescimento da demanda previsto pela empresa foi identificada a necessidade de melhorias na gestão da manutenção. A fim de melhorar a disponibilidade e confiabilidade da linha, foram estabelecidos novos intervalos de manutenções preventivas para os subconjuntos.

0 levantamento e as análises dos MTBFs e MTTRs dos subconjuntos permitiram, além da determinação de intervalos ótimos entre manutenções, a identificação de subconjuntos críticos e a escolha do tipo de ação mais adequada para melhorar seu desempenho. Subconjuntos com baixo MTBF necessitam de melhorias na política de manutenção, subconjuntos com alto MTTR necessitam de melhoria da capacitação e dos recursos da equipe de manutenção.

A política de manutenção proposta neste estudo determina intervalos adequados para a manutenção preventiva através da análise quantitativa dos tempos até a falha de cada subconjunto. A vantagem desse método em comparação com a política corretiva adotada na empresa em estudo, ou com a política de manutenção executada em intervalos idênticos para todos os subconjuntos adotada em muitas empresas é que o ajuste dos intervalos de manutenção evita que equipamentos menos confiáveis falhem com frequência e que equipamentos mais confiáveis sejam revisados desnecessariamente.

A simulação possibilitou a análise da resposta do sistema quanto às horas de parada de máquina e comportamento das horas extras para atender a demanda diária, considerando possíveis variações de demanda, MTBFe MTTR. 0 uso do método de Monte Carlo permitiu a inclusão da variabilidade, natural do 
sistema, na simulação. Esses resultados auxiliam a empresa na tomada de decisão quanto à necessidade e ao tipo de investimentos em manutenção que melhor atenderiam ao cenário de demanda projetado.

Por fim, vale ressaltar as delimitações deste estudo. Este trabalho limitou-se ao estudo de um cenário produtivo em que a demanda deve ser atendida no mesmo turno de trabalho e é possível o uso de horas extras para compensar picos de demanda ou paradas devido a quebra de conjuntos. Outros cenários, por exemplo, em que não é possível o uso de horas extras ou existem estoques que podem amortizar problemas de confiabilidade exigiriam abordagens diferenciadas. 0 cenário com estoque será objeto de um artigo futuro.

\section{Referências}

BEVILACQUA, M.; BRAGLIA, M.; GABRIELLl, R. Monte Carlo Simulation approach for a modified FMECA in a power plant. Quality and Reliability Engineering International, v. 16, p. 313-324, 2000. http://dx.doi.org/10.1002/10991638(200007/08) 16:4<313::AID-QRE434>3.0.CO;2-U

BL00M, N. B. Reliability Centered Maintenance: implementation made simple. New York: McGraw-Hill, 2006.

DESHPANDE, V. S.; MODAK, J. P. Application of RCM to a medium scale industry. Reliability Engineering \& System Safety, v. 77, n. 1, p. 31-43, 2002. http://dx.doi. org/10.1016/S0951-8320(02)00011-X

FARRERO, J. C.; TARRÉS, L. G.; LOSILLA, C. B. Optimization of replacement stocks using a maintenance programme derived from reliability studies of production systems. Industrial Management \& Data Systems, v. 102, n. 4, p. 188196,2002.http://dx.doi.org/10.1108/02635570210423226

FOGLIATTO, F. S.; RIBEIR0, J. L. D. Confiabilidade e Manutenção Industrial. São Paulo: Campus; Elsevier, 2009.

LAFRAIA, J. R. B. Manual de confiabilidade, mantenabilidade e disponibilidade. Rio de Janeiro: Qualitymark, 2001.

MÁRQUEZ, A. C. et al. The maintenance management framework. A pratical view to maintenance management. Journal of
Quality in Maintenance Engineering, v. 15, n. 2, p. 167178, 2009. http://dx.doi.org/10.1108/13552510910961110

MENDES, A. A.; RIBEIRO, J. L. D. Um estudo do suporte quantitativo necessário para a operacionalização da MCC. Produção, v. 21, n. 4, p. 583-593, 2011. http:// dx.doi.org/10.1590/S0103-65132011005000032

NGUYEN, D. Q.; BRAMMER, C.; BAGAJEWICZ, M. New tool for the evaluation of the scheduling of preventive maintenance for chemical process plants. Industrial \& Engineering Chemistry Research, v. 47, n. 6, p. 110-124, 2008. http:// dx.doi.org/10.1021/ie071231i

PINTO, A. K.; XAVIER, J. A. N. Manutenção: função estratégica. 2. ed. Rio de Janeiro: Qualitymark, 2001.

RAO, P. N.; SRIKRISHNA, S.; YADAVA, G. S. Reliability-centred maintenance applied to power plant auxiliaries. Journal of Qualityin Maintenance Engineering, v. 2, n. 1, p. 3-14, 1996. http://dx.doi.org/10.1108/13552519610113809

RAUSAND, M. Reliability Centered Maintenance. Reliability Engineering and System Safety, v. 60, n. 2, p. 121-132, 1998. http://dx.doi.org/10.1016/S0951-8320(98)83005-6

SANTOS, W. B.; COLOSIMO, E. A.; MOTTA, S. B. Tempo ótimo entre manutenções preventivas para sistemas sujeitos a mais de um tipo de evento aleatório. Revista Gestão e Produção, v. 14, n. 1, p. 193-202, 2007. http://dx.doi. org/10.1590/S0104-530X2007000100016

SELLITTO, M. A. Formulação estratégica da manutenção industrial com base na confiabilidade dos equipamentos. Revista Produção, v. 15, n. 1, p. 44-59, 2005.

WILMETH, G. W.; USREY, M. W. Reliability centered maintenance: a case study. Engineering Management Journal, v. 12, n. 4, p. 25-31, 2000.

\section{Agradecimentos}

Agradecemos à Coordenação de Aperfeiçoamento de Pessoal de Nível Superior (Capes) e ao Conselho Nacional de Pesquisa e Desenvolvimento (CNPq) pelo fornecimento de bolsa de pesquisa. Também agradecemos à empresa que permitiu a realização deste estudo colaborando com a disponibilização de informações.

\section{Establishment of a maintenance plan based on quantitative analysis in the context of the MCC in a JIT production scenario} Abstract

This paper presents a method to develop quantitative analysis to support the review or preparation of an equipment maintenance plan in a just-in-time production scenario. The proposed method includes: i) ldentification of parts that influence reliability; ii) Estimation of failure rates; iii) Classification of parts according the effect of their failures; iv) Estimation of line occupation parameters; $v$ ) ldentification of probability distributions for occupation line, time between failures and repair times, vi) Simulation of production and maintenance using the Monte Carlo Method; vii) Accomplishment of a sensitivity analysis concerning variations in demand, $M T B F$ and $M T T R$, and iv) Establishment of maintenance strategy and intervals between preventive maintenance. The method is illustrated through an application comprising the scenario of a labeling and filling gallons line at a paints and dyes production company. The method allowed to clearly identifying the critical parts of the equipment according the production scenario in question.

\section{Keywords}

Reliability centered maintenance. Monte Carlo simulation. Just in time system. Maintenance plan. 